\title{
Lactate Genesis by Rat Liver and Muscle during Development
}

\author{
GEORGE J. DOMBROWSKI, JR. AND KENNETH R. SWIATEK \\ Institute for the Study of Developmental Disabilities [G.J.D., K.R.S.] and Committee on Neuroscience /K.R.S.J. \\ University of Illinois at Chicago, Chicago, Illinois 60608
}

\begin{abstract}
Lactate has been shown to be an important fuel for brain metabolism during early postnatal development (1). In an attempt to identify the source(s) of lactate in the postnatal rat, we have studied the in vitro catabolism of glucose, galactose, fructose, alanine, glycerol, and octanoate in liver and muscle minces prepared from suckling rat pups. Whereas galactose, fructose, and octanoate were found to be lactagenic (lactate generating) in liver, glucose was the sole lactate precursor in muscle. Galactose was most effective as a hepatic lactate source at $3 \mathrm{~d}$ of age. Thereafter, the production of lactate from galactose decreased to reach control levels by $15 \mathrm{~d}$ of age. In contrast, fructose or octanoate were lactagenic throughout development. Lactate formation from galactose was completely halted by iodoacetate, inhibited by high galactose concentrations, and suppressed by fasting. The absence of oxygen increased lactate production from either fructose or octanoate, but it did not affect lactagenesis from galactose. Muscle minces produced lactate from glucose in an agedependent manner similar to the development pattern of lactate formation from galactose by liver. Because lactosederived galactose is readily available during suckling, it is suggested that galactose-based hepatic lactagenesis serves a unique role in maintaining the supply of lactate during early postnatal development. This hepatic capability may augment glucose-based muscle lactate synthesis at a time when lactate is a major brain fuel. (Pediatr Res 30: 331336,1991 )
\end{abstract}

Abbreviations

UDP, uridine diphosphate

Lactate is the major fuel for brain cortex in rat pups during the first postpartum week (1). The utilization of lactate accounts for more than $70 \%$ of cerebral oxidative metabolism in the 6 -dold, normally ventilated rat pup, followed by the consumption of 3-hydroxybutyrate and glucose in approximately equal amounts. The extensive reliance of the young cerebral cortex upon lactate as a fuel prompted this investigation to evaluate the lactagenic' potential of several common metabolites when supplied to liver or muscle during the postnatal period.

Milk, the sole food source available to suckling pups, consists principally of casein, fats, and sugars. Although fats comprise $70-80 \%$ of the caloric content of milk, it is most probable that a sugar provides the carbon for lactate synthesis. Lactose, a disaccharride of glucose and galactose, is the principal milk sugar.

Rcceived February 28. 1991; accepted June 18, 1991.

Correspondence and reprint requests: George J. Dombrowski, Jr. Institute for the Study of Developmental Disabilities, $1640 \mathrm{~W}$. Roosevelt Road, University of Illinois at Chicago. Chicago, IL 60608.

' Lactate generating.
Although glucose-derived muscle lactagenesis is known, there is no information available concerning the relative output of lactate from skeletal muscle as a function of age during the suckling period. In investigations of galactose metabolism during development, the primary emphasis has been the genesis of glucose in fasted animals (2-4). Even though it has been known for many years that the i.v. administration of galactose to infants results in the physiologic elevation of blood lactate levels (5), no attempt has been made to delineate the mechanism of this phenomenon or explain its purpose.

In the present study, we have compared rates of lactagenesis from galactose, glucose, fructose, alanine, glycerol, and octanoate in liver and muscle minces during postnatal development. Several factors have been shown to influence hepatic galactose-based lactate production.

\section{MATERIALS AND METHODS}

Animals and chemicals. Sprague-Dawley rats (15-19 d pregnant) were obtained from Sasco-King Laboratories, Madison, WI. Throughout the remainder of gestation and while nursing rat mothers were fed Purina Chow no. 5012 (Ralston-Purina Co.. St. Louis, MO) ad libitum. Large litters were culled to eight pups within the first $18 \mathrm{~h}$ postpartum. D-Glucose and glycerol were obtained from Fisher Scientific Co., Chicago, IL. D-Fructose was purchased from Calbiochem, Los Angeles, CA. L-alanine was supplied through Eastman Kodak, Rochester, NY. Sodium $n$-octanoate, $\mathrm{D}(+)$-galactose, and the enzymes used for analyses were obtained from Sigma Chemical Co., St. Louis, MO.

All animal experiments were approved by the Animal Care Committee of the University of Illinois at Chicago.

Mince experiments. Normally reared and fed animals at the ages indicated in the figure and table legends were used for all experiments unless otherwise indicated. Either livers or the hind limb skeletal muscles were excised from decapitated rat pups and placed into isotonic saline at $0-4^{\circ} \mathrm{C}$. Muscle tissue was cut from between the knee and foot as well as from between the hip and knee. Because of the small body size of suckling pups, no attempt was made to identify the particular skeletal muscles used for the mince experiments. The soleus, extensor digitorum longus, and gastrocnemius muscles were among those excised. Muscle pieces were trimmed of fat and facia. Tissue minces $(100-160 \mathrm{mg})$ were placed in $25-\mathrm{mL}$ flasks containing $1.8 \mathrm{~mL}$ of Krebs-Henseleit bicarbonate buffer at $\mathrm{pH} 7.4$ (6). Substrates were added as indicated in the table or figure legends, and the final volume was adjusted to $2.0 \mathrm{~mL}$ with water. Stock sodium octanoate $(200$ $\mathrm{mM}$ ) was neutralized with $\mathrm{HCl}$ before it was used. All substrates were tested for the presence of lactic acid and found to be free of contamination. Minces were incubated for $90 \mathrm{~min}$ at $37^{\circ} \mathrm{C}$ in a shaker bath. Unless otherwise indicated, flasks were gassed with $\mathrm{O}_{2} / \mathrm{CO}_{2}(95 \% / 5 \%)$ throughout this period. At the end of the incubation, the racks of flasks were transferred to an ice-water bath and the incubations were terminated by the addition of 0.20 $\mathrm{mL}$ of $3 \mathrm{~N} \mathrm{HClO}_{4}$ to the medium. Flasks were vigorously shaken 
after the addition of the acid. The acidified minces were subsequently homogenized and the mixtures centrifuged at $5000 \times g$ for $20 \mathrm{~min}$. The supernatants $(25-40 \mu \mathrm{L})$ were assayed to determine metabolite levels.

Chemical analyses. Glucose was measured by the coupled glucose oxidase (EC 1.1.3.4)/peroxidase (EC 1.11.1.7) method (7). Contamination of the glucose oxidase/peroxidase system with galactose oxidase was found to be less than $2 \%$ of the glucose reactivity. Lactate was measured with lactic dehydrogenase (EC 1.1.1.27) and $\mathrm{NAD}^{+}$using $100 \mathrm{mM}$ Tris- $\mathrm{HCl}$ buffer at $\mathrm{pH} 8.5$ (8). Iodoacetate had no effect on the determination of lactic acid when present at $1.4 \mathrm{mM}$.

Statistical analyses. Statistical significance was evaluated using the analysis of variance and fixed effect models. Significance was set at $p \leq 0.05$. Unless comparisons were planned, the significance level was appropriately adjusted for multiple comparisons by using a modification of the Bonferroni procedure (9). When the Bonferroni method was applied, it was used within either substrate or age.

\section{RESULTS}

Liver lactate production in postnatal rats. Table 1 lists lactate production when either alanine, galactose, glucose, glycerol, fructose, or octanoate were added to liver minces prepared from rat pups between the ages of 1 and $32 \mathrm{~d}$. Galactose, fructose, and octanoate were found to be lactagenic. Fructose or octanoate produced significantly more lactate than galactose at all ages ( $p$ $<0.03$ ). At 3,12 , and $18 \mathrm{~d}$ of age, octanoate produced significantly more lactate than fructose $(p<0.05)$. Glucose or alanine were ineffective as lactate precursors throughout the suckling period. Glycerol was an ineffective lactate precursor at all ages other than the 12th postnatal day, when a small amount of lactate was formed. Although some significant age-dependent variations in lactate release were observed when either fructose or octanoate were used as substrates, relatively large amounts of lactate were formed from either of these compounds throughout the suckling period as well as at $32 \mathrm{~d}$ of age. In contrast, lactate production from galactose was maximal on the 3 rd postpartum day and galactose was effective as a carbon source for lactate only between the ages of 1 and $12 \mathrm{~d}$. Thereafter, lactate genesis in the presence of this sugar did not differ significantly from that of the control.

Muscle lactagenesis during development. Table 2 lists the amounts of lactate produced when hind limb muscle mince preparations were incubated in the presence of either alanine, galactose, glucose, glycerol, fructose, or octanoate at 6,9 , and 18 d of age. In contrast to the results obtained for liver, glucose was the only substrate effectively catabolized by muscle to yield lactate. Moreover, muscle mince glucose-based lactagenesis was age dependent. Lactate was produced by the muscle obtained from animals at 6 and $9 \mathrm{~d}$ of age, whereas lactate was not produced when the 18-d muscle preparation was incubated with glucose. Similar results were obtained for diaphragm preparations, but a significant age-dependent decrease in lactate production from glucose was not observed with this tissue (results not shown). With diaphragm, small amounts of lactate were also formed from fructose at 9 and $18 \mathrm{~d}$ of age $(0.52 \mu \mathrm{mol} / 100 \mathrm{mg} /$ 90 min).

Characterization of hepatic lactate synthesis. The hepatic capability to produce lactate from fructose has long been recognized $(10,11)$. Moreover, it is expected that galactose-derived lactate is also the result of well-known metabolic pathways. Nevertheless, it is possible for the released lactate to be the product of some other unknown process that may be regulated by the presence of galactose or its catabolites. Additional experiments were conducted to evaluate this possibility.

Purified hepatic galactokinase (EC 2.7.1.6) has been shown to be inhibited by galactose concentrations in excess of $1-2 \mathrm{mM}$ (12). Figure 1 depicts lactate release as a function of either galactose or fructose concentration in liver minces at $9 \mathrm{~d}$ of age. Whereas substrate inhibition was not observed with fructose,

Table 1. Effect of substrate and age on lactate production by minced liver*

\begin{tabular}{|c|c|c|c|c|c|c|c|c|}
\hline \multirow{2}{*}{$\begin{array}{l}\text { Substrate additions } \\
\qquad(10 \mathrm{mM})\end{array}$} & \multicolumn{8}{|c|}{ Age (d) } \\
\hline & 1 & 3 & 6 & 9 & 12 & 15 & 18 & 32 \\
\hline Galactose & $0.49 \dagger$ & $0.81 \dagger$ & $0.42+4$ & $0.58 \dagger$ & $0.43 \dagger$ & $0.09 \ddagger$ & 0.11 & 0.21 \\
\hline SEM & 0.25 & 0.06 & 0.07 & 0.05 & 0.08 & $0.08^{\circ}$ & 0.09 & 0.06 \\
\hline$n$ & 17 & 33 & 33 & 78 & 23 & 18 & 18 & 6 \\
\hline Glucose & 0.20 & 0.00 & -0.02 & 0.11 & $-0.23 \ddagger$ & -0.21 & -0.11 & $0.55+t$ \\
\hline SEM & 0.20 & 0.06 & 0.09 & 0.05 & 0.09 & 0.06 & 0.10 & 0.10 \\
\hline$n$ & 18 & 18 & 12 & 18 & 17 & 18 & 18 & 6 \\
\hline Fructose & $0.94 \dagger$ & $1.19 \dagger$ & $1.10 \dagger$ & $1.51 \dagger$ & $1.03 \dagger t$ & $1.06 \dagger$ & $1.24 \dagger$ & $1.15 \dagger$ \\
\hline SEM & 0.24 & 0.14 & 0.11 & 0.08 & 0.15 & 0.07 & 0.10 & 0.09 \\
\hline$n$ & 18 & 24 & 27 & 42 & 24 & 18 & 18 & 6 \\
\hline L-Alanine & $-0.63 \dagger$ & $-0.15 \ddagger$ & -0.19 & 0.10 & -0.14 & -0.06 & 0.30 & \\
\hline SEM & 0.26 & 0.08 & 0.05 & 0.06 & 0.17 & 0.09 & 0.10 & \\
\hline$n$ & 18 & 12 & 12 & 12 & 11 & 12 & 12 & \\
\hline Glycerol & & 0.20 & 0.07 & 0.12 & $0.30 \dagger$ & 0.09 & 0.09 & 0.34 \\
\hline SEM & & 0.35 & 0.03 & 0.04 & 0.04 & 0.04 & 0.10 & 0.09 \\
\hline$n$ & & 21 & 18 & 21 & 14 & 6 & 6 & 6 \\
\hline Octanoate & & $2.01 \dagger$ & $1.48 t \ddagger$ & $1.18 \dagger$ & $2.11 \dagger \ddagger$ & $1.42 t t$ & $1.82 \dagger$ & $1.16 t t$ \\
\hline SEM & & 0.14 & 0.14 & 0.21 & 0.16 & 0.13 & 0.10 & 0.18 \\
\hline$n$ & & 24 & 27 & 18 & 15 & 6 & 6 & 6 \\
\hline
\end{tabular}

${ }^{*}$ Minces were prepared and incubations performed as described in Materials and Methods. Each value for the added substrates represents the difference between lactate formed in the presence of substrate and lactate released in the absence of added substrate (control). Results are expressed as $\mu \mathrm{mol} / 100 \mathrm{mg}$ mince $/ 90 \mathrm{~min} . n$, number of repetitions. The control means and standard errors were $1.92 \pm 0.24(n=18) .0 .94 \pm 0.09(n=36)$, $0.56 \pm 0.05(n=35), 0.53 \pm 0.03(n=90), 0.80 \pm 0.19(n=24), 0.75 \pm 0.07(n=17), 0.99 \pm 0.11(n=18)$, and $1.71 \pm 0.06(n=6) \mu \mathrm{mol} / 100$ $\mathrm{mg} / 90 \mathrm{~min}$ at $\mathrm{I}, 3,6,9,12,15,18$, and $32 \mathrm{~d}$ of age, respectively. Statistical analyses were performed as described in Materials and Methods. Lactate production was significantly affected by either substrate or age $(p<0.0001)$.

$\uparrow$ Significant change from the age-matched control.

\$ Significant "within substrate" change from the preceding tabulated age. 
Table 2. Effect of substrate and age on lactate release from muscle*

\begin{tabular}{lrcr}
\hline $\begin{array}{l}\text { Substrate } \\
\text { additions } \\
(10 \mathrm{mM})\end{array}$ & \multicolumn{4}{c}{ Age (d) } \\
\hline Glucose & $1.01 \pm 0.13(10)$ & $0.81 \pm 0.09(14)$ & $0.26 \pm 0.13 \dagger(19)$ \\
Galactose & $-0.23 \pm 0.05 \ddagger(4)$ & $0.07 \pm 0.06 \ddagger(8)$ & $-0.23 \pm 0.29 \ddagger(10)$ \\
Fructose & $0.18 \pm 0.07 \ddagger(4)$ & $0.25 \pm 0.11 \ddagger(8)$ & $0.34 \pm 0.16(10)$ \\
l.-Alanine & $-0.03 \pm 0.11 \ddagger(4)$ & $0.06 \pm 0.11 \ddagger(8)$ & $-0.03 \pm 0.20(4)$ \\
Glycerol & $0.23 \pm 0.21 \ddagger(4)$ & $0.24 \pm 0.15 \ddagger(6)$ & $-0.40 \pm 0.39 \dagger(6)$ \\
Octanoate & $0.29 \pm 0.18 \ddagger(5)$ & $0.30 \pm 0.11 \ddagger(7)$ & $0.09 \pm 0.20(6)$ \\
\hline
\end{tabular}

* Hind limb muscle minces were prepared and incubations performed as described in Materials and Methods. Each value represents the 90min mean difference in lactate concentration between incubations performed in the presence of substrate and incubations performed without added substrate (control). Results are expressed as $\mu \mathrm{mol} / 100 \mathrm{mg}$ mince/ $90 \mathrm{~min}$. The control means were $1.03 \pm 0.08(n=9), 1.48 \pm 0.09(n=$ 13), and $2.81 \pm 0.23(n=19) \mu \mathrm{mol} / 100 \mathrm{mg} / 90 \mathrm{~min}$ at 6.9 , and $18 \mathrm{~d}$ of age, respectively. Error is expressed as SEM, and values in parentheses are the number of repetitions. Statistical analyses were performed as described in Materials and Methods. Lactate production was significantly affected by either substrate $(p<0.0001)$ or age $(p<0.021)$.

$\dagger$ Significant change from the preceding age.

$\ddagger$ Significant difference between these results and the age-matched experiments where glucose was added.

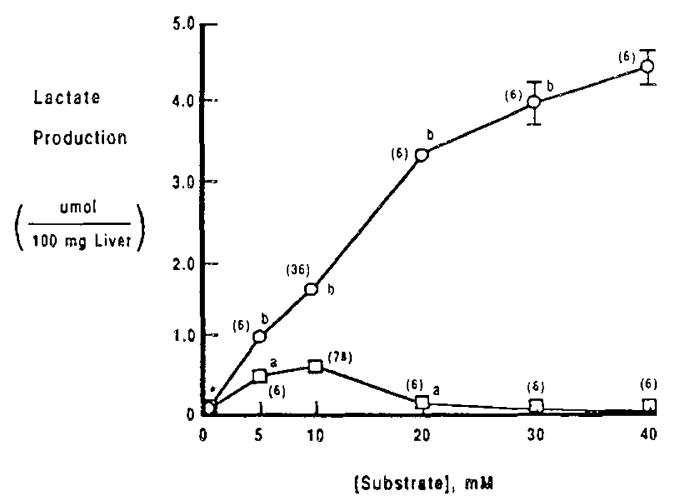

Fig. 1. Lactate production from galactose $(\square)$ and fructose $(O)$ as a function of substrate concentration. Liver minces were prepared at $9 \mathrm{~d}$ of age and incubations were performed as described in Materials and Methods. Each point represents the mean difference in lactate production between incubations performed in the presence of substrate at the indicated concentration and control experiments performed simultaneously without substrate. The control values from each experiment were randomized and subtracted from each other. There were 63 control values for the galactose experiments and 21 control values for the fructose curve. Values in parentheses denote the number of repetitions for all other points. Effects were evaluated as described in Materials and Methods. Substrate or concentration significantly affected lactate production $(p<$ $0.0001)$. Planned comparisons were conducted to test differences in lactate production between neighboring substrate concentrations. $a$, a significant change from the next lower galactose concentration. $b$, a significant change from the next lower fructose concentration. SEM is shown by a set of horizontal bars above and below the plotted point. For points without error bars, the SEM is less than or equal to the height of the plot symbol.

lactate production was inhibited by galactose at concentrations in excess of $10 \mathrm{mM}$. Lactate production from either fructose or octanoate was not decreased in the presence of $40 \mathrm{mM}$ galactose (data not shown). These results suggest that the newly formed lactate is derived from galactose through activation by galactokinase.

Lactate release in the presence of either galactose, fructose, or octanoate was inhibited by the addition of iodoacetate to the liver preparations of 9-d-old animals (Table 3). Although lacta-
Table 3. Effect of iodoacetate on lactate production in liver*

\begin{tabular}{lccc}
\hline $\begin{array}{l}\text { Substrate } \\
\text { additions } \\
(10 \mathrm{mM})\end{array}$ & \multicolumn{2}{c}{$\begin{array}{c}\text { Lactate production }(\mu \mathrm{mol} / 100 \mathrm{mg} \\
\text { mince/90 min })\end{array}$} & $\begin{array}{c}\% \\
\text { Inhibition }\end{array}$ \\
\cline { 2 - 4 } & -Iodoacetate & +Iodoacetate & 100 \\
Galactose & $0.58 \pm 0.05 \dagger(78)$ & $-0.22 \pm 0.07 \pm \$ \|(12)$ & 62 \\
Fructose & $1.51 \pm 0.08 \dagger(42)$ & $0.57 \pm 0.10 \pm \$(12)$ & 32 \\
Octanoate & $1.18 \pm 0.21 \dagger(18)$ & $0.80 \pm 0.10 \ddagger \$(6)$ & \\
\hline
\end{tabular}

* Minces were prepared from rat pups at $9 \mathrm{~d}$ of age, and incubations were performed as described in Materials and Methods. lodoacetate was added as indicated at a concentration of $0.5 \mathrm{mM}$. Error is expressed as SEM, and values in parentheses are the number of repetitions. Statistical analyses were performed as described in Materials and Methods. A change in substrate or the presence of iodoacetate significantly affected lactate production $(p<0.0001)$. Planned comparisons were conducted to test the effect of iodoacetate additions within the substrate factor.

$\dagger$ These values represent the difference between lactate formed in the presence of substrate and lactate released in the absence of additions (control). Mean lactate formation in the control was $0.53 \pm 0.03(n=$ 90) $\mu \mathrm{mol} / 100 \mathrm{mg} / 90 \mathrm{~min}$.

$¥$ These values represent the difference between lactate formed in the presence of substrate + iodoacetate and lactate released in the presence of iodoacetate alone. When iodoacetate was added to the control, mean lactate production was $0.74 \pm 0.09(n=12) \mu \mathrm{mol} / 100 \mathrm{mg} / 90 \mathrm{~min}$.

$\$$ Significant difference between these results and the substratcmatched values where iodoacetate was omitted.

$\|$ Significantly lower value than those obtained from either fructose or octanoate in the presence of iodoacetate.

genesis from galactose was completely halted, lactate formation was only partially suppressed by iodoacetate when either fructose $(62 \%)$ or octanoate $(32 \%)$ were used as substrates. Lactate release in the absence of substrate additions was unaffected by iodoacetate. Galactose-1-phosphate uridyltransferase (EC 2.7.7.12) and UDP-galactose 4-epimerase (EC 5.1.3.2) are known to be sensitive to sulfhydryl reagents $(13,14)$. The $100 \%$ iodoacetate inhibition of lactate formation in the presence of galactose suggests that a metabolic rate-limiting process, such as the galactose-1phosphate uridyltransferase reaction, was affected. Moreover, it is likely for galactose-based lactagenesis to be secondarily affected at another site of iodoacetate susceptibility, 3-phosphoglyceraldehyde dehydrogenase (EC 1.2.1.12). This glycolytic enzyme is pertinent to the catabolism of fructose as well as galactose. The iodoacetate suppression of fructose-based lactate production directly supports the expectation that glycolysis would be involved in lactagenesis during development.

Table 4 lists the effects of oxygen deprivation on lactate formation from alanine, fructose, galactose, glucose, and octanoate in liver minces prepared from pups at $6 \mathrm{~d}$ of age. As expected, in the absence of any added substrate (control), lactate output under the anaerobic condition was significantly higher than that obtained in the presence of oxygen. Nevertheless, significant increases in lactate formation occurred under the anaerobic condition as compared with the paired aerobic experiments when either fructose or octanoate were used as substrates. No significant differences in lactate formation were observed between the aerobic and anaerobic conditions when either galactose, glucose, or alanine were the test substrates. Because neither glucose nor alanine served as lactate precursors in the presence of oxygen, these two substrates were not expected to be lactagenic in the absence of oxygen. However, galactose is an effective lactagenic substrate at $6 \mathrm{~d}$ of age in the presence of oxygen, and, therefore, it could be anticipated that a lack of oxygen would increase the rate of lactate synthesis from this compound. The catabolism of galactose appears to be impaired under anaerobic conditions. This anaerobic effect on lactate formation from galactose may be explained by a decrease in the activity of UDPgalactose 4 -epimerase. This enzyme requires $\mathrm{NAD}^{+}$as a coen- 
Table 4. Lactate formation under aerobic and anaerobic conditions*

\begin{tabular}{|c|c|c|}
\hline \multirow{2}{*}{$\begin{array}{l}\text { Substrate } \\
\text { additions } \\
(10 \mathrm{mM})\end{array}$} & \multicolumn{2}{|c|}{$\begin{array}{l}\text { Lactate production }(\mu \mathrm{mol} / 100 \mathrm{mg} / \\
\qquad 90 \mathrm{~min})\end{array}$} \\
\hline & Aerobic & Anaerobic \\
\hline Galactose & $0.42 \pm 0.07(33)$ & $0.30 \pm 0.17(15)$ \\
\hline Glucose & $-0.02 \pm 0.09(12)$ & $0.19 \pm 0.25(5)$ \\
\hline Fructose & $1.10 \pm 0.11(27)$ & $1.58 \pm 0.26+(15)$ \\
\hline I-Alanine & $-0.19 \pm 0.05(12)$ & $0.15 \pm 0.34(6)$ \\
\hline Octanoate & $1.48 \pm 0.14(27)$ & $2.10 \pm 0.36+(13)$ \\
\hline
\end{tabular}

* Liver minces were prepared on the 6th postnatal day, and incubations were performed as described in Materials and Methods. A gas mixture of $\mathrm{O}_{2} / \mathrm{CO}_{2}(95: 5)$ was used to aerate the aerobic tests, and a mixture $\mathrm{N}_{2} / \mathrm{CO}_{2}(95: 5)$ was used for the anaerobic experiments. Values are expressed as the mean difference in lactate formation between incubations performed with substrate and control experiments performed simultaneously without substrate. Mean control lactate formation in the absence of added substrate was $0.56 \pm 0.06(n=35)$ and $2.18 \pm 0.23(n$ $=15) \mu \mathrm{mol} / 100 \mathrm{mg} / 90 \mathrm{~min}$ for the aerobic and anaerobic conditions, respectively. Error is expressed as SEM, and values in parentheses indicate the number of repetitions. Statistical analyses were performed as described in Materials and Methods. Changes in substrate or the absence of oxygen significantly affected lactate production $(p<0.0001)$. Planned comparisons were conducted between the aerobic and anaerobic conditions.

+ Significant difference between the aerobic and anaerobic conditions $(p<0.05)$

Table 5. Effect of fasting on lactate production by liver*

\begin{tabular}{|c|c|c|}
\hline \multirow{2}{*}{$\begin{array}{l}\text { Age and } \\
\text { treatment }\end{array}$} & \multicolumn{2}{|c|}{ Substrate $(10 \mathrm{mM})$} \\
\hline & Galactose & Fructose \\
\hline \multicolumn{3}{|l|}{$3 \mathrm{~d}$ old } \\
\hline Fed & $0.81 \pm 0.06(33)$ & $1.19 \pm 0.14 \uparrow(24)$ \\
\hline Fasted & $0.14 \pm 0.03 \div(18)$ & $0.28 \pm 0.05 \neq(18)$ \\
\hline \multicolumn{3}{|l|}{$9 \mathrm{~d}$ old } \\
\hline Fed & $0.58 \pm 0.05 \$(78)$ & $1.51 \pm 0.08+(42)$ \\
\hline Fasted & $0.37 \pm 0.06 \div(24)$ & $0.79 \pm 0.08+ \pm \$(24)$ \\
\hline
\end{tabular}

* Rat pups were fasted by separating them from their mothers for a period of $18 \mathrm{~h}$ immediately before the experiment. During the fast, pups were maintained in a plastic bowl equipped with absorbent bedding and floated on a water bath at $37^{\circ} \mathrm{C}$. Minces were prepared and incubations performed as described in Materials and Methods. Each value represents the mean difference between lactate formed in the presence of substrate and lactate released in the absence of added substrate (control). Results are expressed as $\mu \mathrm{mol} / 100 \mathrm{mg}$ tissue $/ 90 \mathrm{~min}$. Control means for the fed animals were $0.94 \pm 0.09(n=36)$ and $0.53 \pm 0.03(n=90) \mu \mathrm{mol} / 100$ $\mathrm{mg} / 90 \mathrm{~min}$ at 3 and $9 \mathrm{~d}$ of age, respectively. The control means for the fasted animals were $0.26 \pm 0.03(n=18)$ and $0.20 \pm 0.02(n=24) \mu \mathrm{mol} /$ $100 \mathrm{mg} / 90 \mathrm{~min}$ at 3 and $9 \mathrm{~d}$ of age, respectively. Error is expressed as SEM, and the values in parentheses indicate the number of repetitions. A three-factor statistical analysis was performed as described in Materials and Methods. Lactate production was significantly affected by either substrate $(p<0.0001)$, age $(p<0.0012)$, or fasting $(p<0.0001)$. Planned comparisons were conducted between fed and fasted conditions.

+ Significant difference in lactate formation between incubations where galactose was the substrate and incubations where fructose was the substrate, age and treatment held constant.

$\ddagger$ Significant difference between the fed and fasted values with age and substrate matched.

$\S$ Significant age-related effect with all other conditions constant.

zyme, and a lack of oxygen decreases the level of oxidized pyrimidine dinucleotide.

Fasting and lactate production. An 18-h fast significantly decreased hepatic lactate formation from either galactose or fructose, and the effect was age dependent (Table 5). Fasting caused a $76-83 \%$ reduction in lactate synthesis at $3 \mathrm{~d}$ of age, whereas only a $36-48 \%$ decrease was observed for the liver minces of fasted 9-d-old pups. Fasting also significantly decreased lactate release in the control incubations performed without added substrate at these same ages.

Galactose supplementation of the suckling diet has previously been shown to enhance the activity of galactose-catabolizing enzymes in intestine (15) as well as in liver (16). To determine whether the decreased lactate formation of fasted animals was the result of a decreased glycolytic rate or the altered activity of Leloir enzymes plus phosphoglucomutase (EC 5.4.2.2) or fructokinase (EC 2.7.1.4) plus aldolase-B (fructose-1-phosphate aldolase, EC 4.1.2.13), glucose production was also monitored in these experiments (Table 6). Fasting did not affect glucose synthesis from either galactose or fructose at $3 \mathrm{~d}$ of age. However, glucose formation from either substrate was significantly enhanced by fasting in the 9 -d-old pups. Fasting does not appear to decrease the activity of either galactokinase, the Leloir pathway, phosphoglucomutase, or fructokinase/aldolase-B enzymes and may even enhance their respective capacities. The fastingrelated decrease in lactate output (Table 5) is most likely the result of decreased glycolytic rate.

\section{DISCUSSION}

Lactate has been demonstrated to be a major brain cortex fuel in normally reared rats during the neonatal period (1). Our results indicate that liver and muscle are principal sites of lactate production during early postnatal development. We have demonstrated that, whereas glucose is the sole lactate precursor in muscle, suckling liver can produce lactate from either galactose, fructose, or octanoate, but not from glucose. Both hepatic galactose-based lactate synthesis and muscle glucose-based lactate production were found to be similarly age dependent. In each case, the period of peak lactagenesis corresponds to the first 9 postnatal days (Tables 1 and 2).

The age-related pattern of liver lactate formation from galactose parallels the postnatal hepatic activities of galactokinase (12,

Table 6. Effect of fasting on glucose production by liver*

\begin{tabular}{ccc}
\hline \multirow{2}{*}{$\begin{array}{c}\text { Age and } \\
\text { treatment }\end{array}$} & \multicolumn{2}{c}{ Substrate $(10 \mathrm{mM})$} \\
3 d old & & Fructose \\
$\quad$ Fed & $2.26 \pm 0.34(33)$ & $2.72 \pm 0.35(24)$ \\
Fasted & $2.82 \pm 0.32(18)$ & $4.07 \pm 0.34(18)$ \\
9 d old & & \\
Fed & $3.04 \pm 0.37(60)$ & $4.25 \pm 0.51(36)$ \\
Fasted & $3.82 \pm 0.51 \dagger(24)$ & $6.19 \pm 0.41 \dagger \$ \S(24)$ \\
\hline
\end{tabular}

* Rat pups were maintained and fasted as described in the footnotes to Table 5. Minces were prepared and incubations performed as described in Materials and Methods. Each value represents the mean difference between glucose formed in the presence of substrate and glucose released in the absence of added substrate (control). Results are expressed as $\mu \mathrm{mol} / 100 \mathrm{mg}$ tissue $/ 90 \mathrm{~min}$. Control means for the fed animals were $5.17 \pm 0.19(n=36)$ and $6.57 \pm 0.35(n=66) \mu \mathrm{mol} / 100 \mathrm{mg} / 90 \mathrm{~min}$ at 3 and $9 \mathrm{~d}$ of age, respectively. The control means for fasted animals were $1.91 \pm 0.21(n=18)$ and $2.28 \pm 0.25(n=24) \mu \mathrm{mol} / 100 \mathrm{mg} / 90 \mathrm{~min}$ at 3 and $9 \mathrm{~d}$ of age, respectively. Error is expressed as SEM, and the values in parentheses indicate the number of repetitions. A three-factor statistical analysis was performed as described in Materials and Methods. Glucose production was significantly affected by either substrate $(p<$ $0.0005)$, age $(p<0.0033)$, or fasting $(p<0.0005)$. Planned comparisons were conducted between the fed and fasted conditions.

$\dagger$ Significant difference between the fed and fasted values with age and substrate matched $(p \leq 0.03)$.

$\$$ Significant age-related effect with all other conditions constant.

$\S$ Significant difference in glucose formation between incubations where galactose was the substrate and incubations where fructose was the substrate, age and treatment held constant. 
17), galactose-1-phosphate uridyltransferase (13), and UDP-galactose 4-epimerase (14), as well as the age dependency of ${ }^{14} \mathrm{CO}_{2}$ release from hepatocytes incubated with ${ }^{14} \mathrm{C}$-galactose (18). Galactose-based lactate synthesis was inhibited by high levels of galactose (Fig. 1) and halted by iodoacetate (Table 3). Moreover, lactate synthesis from galactose was unaffected by the absence of oxygen, whereas the absence of oxygen increased lactate formation from either fructose or octanoate (Table 4). These results suggest that galactose serves as the carbon source of the generated lactate and, furthermore, that the lactate is formed via enzymes of the Leloir pathway and glycolysis.

A previous report (2) did suggest that galactose-dependent lactate formation may have been age sensitive in hepatocytes obtained from fasted rats $7 \mathrm{~d}$ of age and older. We have shown that fasting sharply decreases lactate production from either galactose or fructose (Table 5) and increases glucose formation from either galactose or fructose (Table 6).

It is surprising that the liver of fed suckling rats did not convert glucose to lactate, although it was formed from galactose (Table 1). Because both glucose and galactose enter glycolysis by way of glucose-6-phosphate, one might suspect that the rate of glucose phosphorylation by hexokinase (EC 2.7.1.2) and glucokinase (EC 2.7.1.2) may be rate limiting. Although these enzyme levels are low during the first $2 \mathrm{wk}$ after birth (19), their total activity is similar to that reported for galactokinase $(20,21)$ during this same period. The possibility exists that the glucose-6-phosphate derived by the hexokinase-glucokinase reaction is hydrolyzed back to glucose as fast as it is formed through the action of the enzyme glucose-6-phosphatase (EC 3.1.3.9), which reaches its highest activity between 3 and $9 \mathrm{~d}$ after birth (21). When glucose6-phosphate was added to the liver preparations at $10 \mathrm{mM}$, large amounts of glucose were generated but lactate was not formed (results not shown). Despite these observations, we did find that galactose produced lactate when incubated with these same liver preparations. These results suggest that the glucose-6-phosphate formed from galactose may not be metabolically equivalent to that derived from glucose, and it is our conclusion that this effect may be due to the compartmentation of glucose-6-phosphate, resulting in a differential exposure of glucose-6-phosphate to the enzyme glucose-6-phosphatase.

In contrast to the results we obtained for galactose, fructose produced lactate at all ages studied with liver minces (Table 1). Hepatic fructose utilization proceeds via the enzymes fructokinase and aldolase-B (22). Fructokinase specifically phosphorylates fructose at position one, and aldolase-B cleaves the product to dihydroxyacetone phosphate plus D-3-glyceraldehyde. Lactate production from fructose during development is well correlated with the activity of aldolase- $B$ during suckling, but it does not parallel the 2.8-fold increase in fructokinase levels between birth and the 18th postpartum day (23-25). These enzymes afford fructose an alternative route to glycolytic trioses that bypasses regulation by the enzyme 6-phosphofructokinase (EC 2.7.1.11). Despite the glycolytic regulation by 6-phosphofructokinase often observed, lactate was formed from galactose even though its carbons are injected into glycolysis at a point before 6-phosphofructokinase (Table 1). The generation of lactate from galactose appears to depend upon the activities of galactokinase, UDPgalactose uridyltransferase, UDP-galactose 4-epimerase, and phosphoglucomutase and appears not to be limited by the hepatic activity of 6-phosphofructokinase.

Octanoate was lactagenic in liver throughout development, but not in muscle (Tables 1 and 2). At this time, we have no explanation as to why octanoate is such a good precursor for hepatic lactate synthesis. It was considered that octanoate at a concentration of $10 \mathrm{mM}$ may have damaged the mitochondria of the liver preparations, concomitantly impairing respiration. However, neither ketogenic nor gluconeogenic capabilities, mitochondrially dependent processes, were affected (data not shown). The hepatic capability to produce lactate from longchain fatty acids such as those derived from milk triglycerides may differ from the results obtained with octanoate. Lactate was also not formed in the presence of $20 \mathrm{mM}$ DL-3-hydroxybutyrate (results not shown).

Skeletal muscle from young animals was significantly better at converting added glucose to lactate than the muscle obtained from 18-d-old pups (Table 2). One reason for the decrease in muscle glucose-based lactate synthesis between 9 and $18 \mathrm{~d}$ of age may be the increase in the level of glucose 1,6-diphosphate, a known inhibitor of hexokinase (26). Another reason for this decreased glucose-based lactate synthesis may be the increased ability to utilize muscle glycogen stores. Glycogen synthesis and its utilization as an alternate substrate can be correlated with the increased enzyme activities of both glycogen synthetase (EC 2.4.1.11) and phosphorylase (EC 2.4.1.1). Indeed, we observed that, in control experiments in which glucose was not added to the incubation mixtures, significant amounts of lactate were formed (Table 2). Glucose 1,6-diphosphate can also increase muscle glycogen mobilization through its stimulation of the glycolytic enzymes phosphoglucomutase and 6-phosphofructokinase (26). Another developmental change occurring at this time is the increased contractile activity of rat hind limb muscle, which requires additional energy that can be supplied in part by glycogen mobilization and its metabolism. During the first $8 \mathrm{~d}$ of life, rat pups are relatively inactive (27) and glucose metabolism to lactate in muscle can be reserved in part to meet the high rate of lactate utilization by rat brain during this time (1).

Finally, although the genesis of lactate in minced tissue preparations may differ from its production by whole organs in vivo, the results of these studies provide some reasons why lactose is the predominant carbohydrate component of rat milk. Lactose is hydrolyzed in the rat intestine to glucose and galactose and absorbed by the mysenteric circulation and delivered to liver via the portal vein. Galactose has been shown to be removed much more efficiently by the liver from the circulation than glucose (28). These data suggest that galactose-based hepatic lactate synthesis augments glucose-based lactate generation in muscle during suckling to provide lactate fuel for metabolism by the newborn rat brain (1)

Acknowledgment. The authors thank Jocelyn Marrow for her invaluable technical assistance.

\section{REFERENCES}

1. Dombrowski Jr GJ, Swiatek KR. Chao KL 1989 Lactate 3-hydroxybutyrate and glucose as substrates for the early postnatal rat brain. Neurochem Res $14: 667-675$

2. Rogers S, Guerra M, Segal S 1983 Galactose metabolism in suckling adult and isolated rat hepatocytes. Pediatr Res 17:609-616

3. Fukushima N. Khallas-Cohen M. Kalant N 1981 Galactose and glucose metabolism by cultured hepatocytes: responsiveness to insulin and the effect of age. Dev Biol 84:359-363

4. Berman WF, Rogers SR, Bautista JO, Segal S 1978 Galactose and glucose metabolism in the isolated perfused suckling and adult rat liver. Metabolism 27:1721-1731

5. Comblath M. Wybregt SH. Bacns GS 1963 Studies of carbohydrate metabolism in the newborn infant. VII. Tests of carbohydrate tolerance in the premature infants. Pediatrics 32:1007-1024

6. Krebs HA Henseleit K 1932 Untersuchungen uber die harnstoffbildung im tierkorper. Hoppe-Scyler's Z Physiol Chem 210:33-66

7. Bergmeyer HU, Bernt E 1974 D-Glucose. Determination with glucose oxidase and peroxidase. In: Bergmeyer HU (ed) Methods of Enzymatic Analysis. Academic Press, New York, pp 1205-1212

8. Lowry OH, Passoneau JV 1972 A collection of metabolite assays. In: A Flexible System of Enzymatic Analysis. Academic Press, New York, pp 147-217

9. Hommel G 1988 A stagewise rejective multiple test procedure based on a modified Bonferroni test. Biometrika 75:383-386

10. Reiser S 1987 Uric acid and lactic acid. In: Reiser S. Hallfrisch J (cds) Metabolic Effects of Dictary Fructose. CRC Press. Boca Raton. Florida, pp 113-134

11. Bantle JP. Laine DD. Thomas JW 1986 Metabolic effects of dietary fructose and sucrose in types I and II diabetic subjects. J Am Med Assoc 256:32413246

12. Walker DG, Khan HH 1968 Some propertics of galactokinase in developing rat liver. Biochem J 108:169-175

13. Bertoli D. Segal S 1966 Developmental aspects and some characteristics of mammalian galactose 1-phosphate uridyltransferase. J Biol Chem 241:40234029 
14. Cohn R, Segal S 1968 Some characteristics and developmental aspects of rat uridine diphosphogalactose 4-epimerase. Biochim Biophys Acta 171:333341

15. Stifel FB, Herman RH, Rosensweig NS 1968 Dietary regulation of galactosemetabolizing enzymes: adaptive changes in rat jejunum. Science 162:692693

16. Rogers SR, Bovee BW, Saunders SL. Segal S 1989 Galactose as a regulatory factor of its own metabolism by rat liver. Metabolism 38:810-815

17. Walker DG. Khan HH, Eaton SW 1965-66 Enzymes catalyzing the phos phorylation of hexoses in neonatal animals. Biol Neonat 9:224-239

18. Segal S, Roth H, Bertoli D 1963 Galactose metabolism by rat liver tissue: influence of age. Science 142:1311-1313

19. Dileepan KN, Wagle SR, Hofman F, Decker K 1979 Distribution profile of glucokinase and hexokinase in parenchymal and sinusoidal cells of rat liver during development. Life Sci 24:89-96

20. Cuatrecasas P, Segal S 1965 Mammalian galactokinase. Developmental and adaptive characteristics in the rat liver. J Biol Chem 240:2382-2388

21. Burch HB, Lowry OH, Kuhlman AM, Skerjance J, Diamant EJ, Lowry SR,
Von Dippe P 1963 Changes in patterns of enzymes of carbohydrate metabolism in the developing rat liver. J Biol Chem 238:2267-2273

22. Hallfrisch J 1990 Metabolic effects of dietary fructose. FASEB J 4:2652-2660

23. Sillero A, Sillero MAG, Sols A 1970 Development of the enzymes of fructose and glyceraldehyde metabolism in liver. Enzymol Biol Clin 11:563-566

24. Hommes FA, Wilmink CW 1968 Developmental changes of glycolytic enzymes in rat brain, liver and skeletal muscle. Biol Neonat 12:181-193

25. Walker DG 1963 The post-natal development of hepatic fructokinase. Biochem J $87: 576-581$

26. Beitner R, Nordenberg J, Cohen TJ 1979 Correlation between the levels of glucose 1,6-diphosphate and the activities of phosphofructokinase, phosphoglucomutase, and hexokinase, in skeletal and heart muscles from rats of different ages. Int J Biochem 10:603-608

27. Kendrick-Jones J, Perry SV 1967 The enzymes of adenine nucleotide metabolism in developing skeletal muscle. Biochem J 103:207-214

28. Goresky CA, Bach GG, Nadeau BE 1971 On the uptake of materials by the intact liver. The transport and net removal of galactose. J Clin Invest 52:9911009 